\title{
An Enhanced Palm Vein Recognition System Using Multi-level Fusion of Multimodal Features and Adaptive Resonance Theory
}

\author{
M.Deepamalar and M.Madheswaran \\ Centre for Advanced Research, Electronics and Communication Engineering \\ Muthayammal Engineering College, Rasipuram, Tamilnadu, India
}

\begin{abstract}
An improved palm vein recognition system using multimodal features and neural network classifier has been developed and presented in this paper. The effects of fusion of multiple features at various levels have been demonstrated. The shape and texture features have been considered for recognition of authenticated users and it is validated using neural network classifier. The recognition accuracy of the proposed system has been compared with the existing techniques. It is found that the recognition accuracy is $99.61 \%$ when the multimodal features fused at matching score level. This proposed multimodal palm vein recognition system is expected to provide reliable security.
\end{abstract}

\section{Categories and Subject Descriptors G4. MATLAB}

\section{General Terms}

Experimentation

\section{Keywords}

Palm vein recognition, Multimodal Biometrics, Feature subset selection, ASFFS, FAR, FRR

\section{INTRODUCTION}

In the past decade there has been a research concerning Unimodal analysis of palm vein identification. Unimodal palm vein identification can be concerned with a variety of problems such as noisy data, intra-class variations, restricted degrees of freedom, non-universality, spoof attacks and unacceptable error rates [1-2,5].

The "Laplacian" multimodal presented by Jain-Gang Wang et al [3] and The "Laplacian" and "Junction Point" multimodal presented by Jain-Gang Wang et al [4] have been based on fusion at imaging level. One of the major issues in imaging fusion is image alignment or registration, which refer to pixel - by - pixel alignment of the images. The proposed palm vein recognition system is expected to overcome some of the limitations of the existing work.

\section{MULTMODAL PALM VEIN RECOGNITION SYSTEM}

Palm vein pattern recognition by a computer can be described as a transformation from the measurement space $\mathrm{M}$ to the decision space $\mathrm{D}$. The mapping function $M \rightarrow D$ is described by the extended transition function of $\delta(M, D)$. This extended transition function $\delta$ can be expanded as $\delta(\delta(\delta(M, F), O), D)$

where the elements of $\mathrm{M}$ are preprocessed palm vein image, the elements of $f \in F$ are multiple features set, the elements of $o \in O$ are optimized feature set and the elements $d \in D$ are termed as decision.

This proposed multimodal system can be developed by fusion of biometric units in two levels such as feature extraction level and decision level which can be used to improve the recognition accuracy. Level 1 fusion can be done at feature extraction level by convolving the hand shape feature and texture of the palm. Level 2 fusion can be done at decision level by combining the decisions based on the matching module can be constructed by Adaptive Resonance Theory (ART1) of convolved multiple feature set, skeleton, bifurcation and ending points of the palm vein image.

\section{PALM VEIN IMAGING AND PREPROCESSING}

The photographs of the vein image are in poor contrast due to glare, and contains irregular shading caused by various thicknesses of skin and bones. Vein pattern authentication requires a normalized and enhanced vein image to authenticate a reliable user. This paper presents a de-noising and enhancement technique based on GSZ - shock filter, which focuses on both noise elimination and edge enhancement. This approach is an edge preserving technique based on 
non-linear diffusion, in which the image gradient has been used to weight the diffusion process in order to smooth the vein image while preserving the vein edge. Guy Gilboa, Nir Sochen, and Yehoshua Y.Zeevi introduce complex Shock filters. They derive the complex shock filter formulation for small $\theta[7-9]$.

$$
I_{t}=-\left(\frac{2}{\pi}\right) \arctan \left(\alpha I_{m}\left(\frac{I}{\theta}\right)\right)|\nabla I|+\lambda I_{x x}
$$

Where $\lambda=\operatorname{rei} \theta$ is a complex scalar. Generalization of the complex shock filter to $2 \mathrm{D}$ yields:

$$
I_{t}=-\left(\frac{2}{\pi}\right) \arctan \left(\alpha I_{m}\left(\frac{I}{\theta}\right)\right)|\nabla I|+\lambda I_{\eta \eta}+\lambda^{\sim} I_{\xi \xi}
$$

where $\lambda \sim$ is a scalar. Binarization is the ease of segmenting the image into two levels object and background.

\section{MULTIPLE FEATURE EXTRACTION}

Multiple Feature extraction technique extracts hand shape features, which is convolved with texture feature to get the multiple feature set, this process is called as fusion at feature extraction level. This phase also extracts skeleton, bifurcation and ending points of the palm vein image [5-6]

\subsection{Fusion at extraction level}

Shape of the palm is extracted by the palm size $\|\mathrm{A} 1 \mathrm{~A} 2\|$ and the distance between $\mathrm{O} 1$ and $\mathrm{O} 2$. These values are constant according to the hand size, determined by the hand width computed by the Euclidean distance between the points $\mathrm{X}$ [L1] and X [L2]. Where L1 and L2 are fixed indexes obtained by trial and error basis.

$$
\begin{aligned}
& \|\mathrm{O} 1 \mathrm{O} 2\|=\alpha\|\mathrm{X}[\mathrm{L} 1] \mathrm{X}[\mathrm{L} 2]\| \\
& \|\mathrm{A} 1 \mathrm{~A} 2\|=\beta\|\mathrm{X}[\mathrm{L} 1] \mathrm{X}[\mathrm{L} 2]\|
\end{aligned}
$$

where $\alpha$ and $\beta$ are the dimensions coefficient. Then the palm size is resized with the fixed size $M^{*} \mathrm{M}$, where $\mathrm{M}$ is as 100 .

The texture features can be obtained using Gabor wavelet. Normally the Gabor features provide the visual properties such as the spatial location, orientation selectivity, and spatial frequency characteristics. The Gabor wavelets defined as follows:

$G_{u, v}(z)=\left(\left\|k_{u, v}\right\|^{2} / \sigma^{2}\right) e^{\left(-\left\|k_{u, v}\right\|^{2}\|z\|^{2} / 2 \sigma^{2}\right)}\left[e^{i k_{u, v}{ }^{*} z}-e^{-\sigma^{2} / 2}\right]$

where

$$
k_{u, v}=\left(\begin{array}{l}
k_{v} \cos \Phi_{u} \\
k_{v} \sin \Phi_{u}
\end{array}\right), k_{v}=\frac{f_{\max }}{2^{v+2}}, \Phi_{u}=u \frac{\pi}{8}, v
$$

$\mathrm{v}$ is the scale of the kernal and $\mathrm{u}$ is the orientation with $\mathrm{f}$ (spatial frequency fixed at 1.26), $\Phi$ controls the function orientation, $\sigma$ is the standard deviation of the Gaussian factor and $\mathrm{z}=(\mathrm{x}, \mathrm{y})^{\mathrm{T}}$.

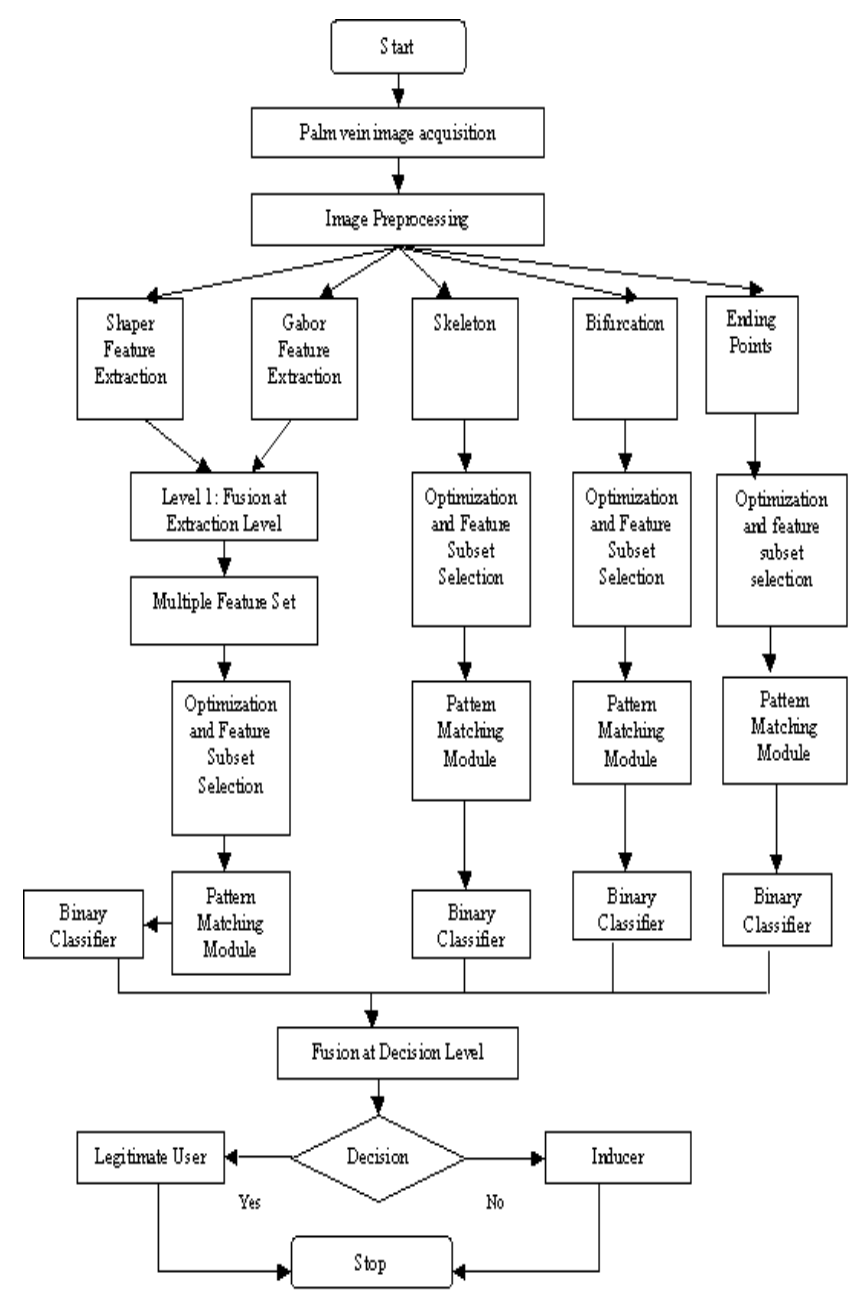

Figure 1. Flow graph of multimodal

The Gabor wavelets can be used to enhance the features in certain scales and orientations. Let $\mathrm{Gu}, \mathrm{v}(\mathrm{Z})$ denotes the Gabor magnitude feature of an image, where $\mathrm{u}$ and $\mathrm{v}$ are the orientation and scale of the kernel $(\mathrm{k})$, respectively. The feature extraction is based on the multi-region mean and Gabor magnitude feature and can be represented as [4]

$\overline{G \mu}_{u, v, l}=\frac{1}{N} \sum_{Z_{i}=R_{l}} G_{u, v}\left(Z_{j}\right)$

where $1=1,2, \ldots . \mathrm{L}$ is the region index, and $\mathrm{L}$ is the number of sub-region. $\mathrm{N}$ represents the total number of pixels in the region Rl. 

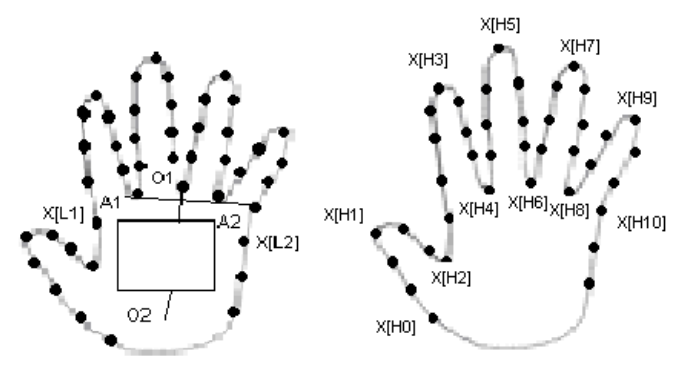

Figure 2. Hand Shape Features

The palm features are obtained by the convolution result of the palm vein input image I with the Gabor filter by [10]

$\mathrm{C}(\mathrm{x}, \mathrm{y})=\mathrm{I}(\mathrm{x}, \mathrm{y}) * \mathrm{G} \mathrm{u}, \mathrm{v}(\mathrm{Z})$

Where $*$ is the convolution operator. The distance between the dominant points, which are defined by, approximately calculates the extracted hand shape characteristics such as widths and lengths of the finger.

$$
\begin{aligned}
& \mathrm{L}[\mathrm{i}]=\mathrm{d}(\mathrm{m}(\mathrm{X}[\mathrm{H} 2 \mathrm{i}], \mathrm{X}[\mathrm{H} 2 \mathrm{i}+2]), \mathrm{X}[\mathrm{H} 2 \mathrm{i}+1]) \\
& 1[\mathrm{i}][\mathrm{j}]=\mathrm{d}(\mathrm{X}[\mathrm{H} 2 \mathrm{i}+1-\mathrm{j}], \mathrm{X}[\mathrm{H} 2 \mathrm{i}+1+\mathrm{j}])
\end{aligned}
$$

where $d(a, b)$ is the Euclidean distance between the points a and $b, m(a, b)$ returns the medium of the segment $\|a b\|, L[i]$ is the $i^{\text {th }}$ finger length, $1[i][j]$ is the $\mathrm{j}^{\text {th }}$ width of the $i^{\text {th }}$ finger and $\mathrm{Hi}=\mathrm{i}(\mathrm{N}+\mathrm{l})$ is the index of the $\mathrm{i}^{\text {th }}$ initial characteristics point of the hand, which are shown in figure 2 .

The fusion of palm vein texture obtained by Gabor filters and hand shape features is done by the convolution. The result of the convolution is binarized and the process is defined by[10$11]$

$\mathrm{F}(\mathrm{x}, \mathrm{y})=\mathrm{b}(\mathrm{C}(\mathrm{x}, \mathrm{y}) * \mathrm{H}(\mathrm{x}, \mathrm{y}))$

Where $\mathrm{b}(\mathrm{x})=0$ if $\mathrm{x}<0$ and $\mathrm{b}(\mathrm{x})=1$ otherwise, $*$ is the convolution operator and $\mathrm{H}$ is the $5 \mathrm{X} 5$ filter based on the share feature. $\mathrm{H}$ filter is defined by:

$$
H(x, y)=H^{\prime}(x, y) \times \frac{1}{\sum_{i=0}^{4} \sum_{j=0}^{4} H^{*}[i \mathrm{I} j]}
$$

Where $\mathrm{H}^{\prime}$ (The normalization coefficient) =

\begin{tabular}{|l|l|l|l|l|}
\hline $\mathrm{L}[0]$ & $1[0][1]$ & $1[0][2]$ & $1[0][3]$ & $1[0][4]$ \\
\hline $\mathrm{L}[1]$ & $1[1][1]$ & $1[1][2]$ & $1[1][3]$ & $1[1][4]$ \\
\hline $\mathrm{L}[2]$ & $1[2][1]$ & $1[2][2]$ & $1[2][3]$ & $1[2][4]$ \\
\hline $\mathrm{L}[3]$ & $1[3][1]$ & $1[3][2]$ & $1[3][3]$ & $1[3][4]$ \\
\hline $\mathrm{L}[4]$ & $1[4][1]$ & $1[4][2]$ & $1[4][3]$ & $1[4][4]$ \\
\hline
\end{tabular}

\subsection{Skeleton Extraction}

A palm vein pattern can be well represented by its skeleton. The skeleton of the vein pattern is extracted by the thinning and pruning algorithm based on local threshold and local mean value. $[4,10]$

$\mu_{i, j}=\left(\frac{1}{m} \times n\right) \sum_{i}^{m} \sum_{j}^{n} I(i, j)$

where $\mathrm{m}$ and $\mathrm{n}$ are the indices of local rectangular region.

$I(i, j)=\left\{\begin{array}{c}1, i f l(i, j) \geq\left(\mu_{i, j}-T_{\mathrm{g}}\right) \\ 0, \text { otherwise }\end{array}\right.$

\subsection{Bifurcation and Ending points extraction}

Palm vein patterns contained two main features, ending and bifurcation. The endings are the end points of a thinned line and the bifurcation is the junction point of three lines. If the current pixel in the skeleton feature is $\left(\mathrm{x}_{\mathrm{c}}, \mathrm{y}_{\mathrm{c}}\right)$ and then its neighboring pixels can defined as $\mathrm{N}_{\mathrm{r}}\left(\mathrm{x}_{\mathrm{c}}, \mathrm{y}_{\mathrm{c}}\right)$ in a $3 \mathrm{X} 3$ matrix.

$N_{c}=T_{c} \cap N_{r}\left(x_{c}, y_{c}\right)$

where $\mathrm{N}_{\mathrm{c}}$ is the number of neighboring pixels of $\left(\mathrm{x}_{\mathrm{c}}, \mathrm{y}_{\mathrm{c}}\right), \mathrm{T}_{\mathrm{c}}$ is the pixel set available in skeleton feature.

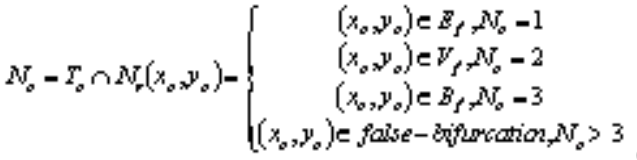

where $B_{f}$ is a set of bifurcations, $E_{f}$ is a set of ending points and $V_{f}$ is a set of pixel in the vein path $[4,10]$.

\section{FEATURE OPTIMIZATION}

The number of features used for recognition is more, and then the network becomes unbelievably complex, which may increase the size of the network, the training time, the set size and the classification time. It is therefore necessary to reduce the number of features for maintaining the acceptable accuracy.

Adaptive sequential floating forward search (ASFFS) is one of the best feature selection methods. Most significant feature can be added with current subset and the least significant feature can be conditionally removed from the current subset iteratively by this method. ASFFS starts from the empty set. After each forward adding step, it performs backward conditional removing steps as long as the objective function $(\mathrm{Az})$ of the current subset increases. In this paper every feature set such as fused set of hand shape and texture, skeleton, bifurcation and ending points can be optimized by the ASFFS optimization technique [12]. 


\section{PALM VEIN PATTERN MATCHING}

The kNN classifier applied for recognition can compare the unknown palm vein pattern that can be the training pattern with the database, which consist of the palm vein pattern of registered users. Hence this approach is lazy learning or memory based learning approach, which can be lead to increase the computational complexity and cost during the testing process. The proposed palm vein system can be done the pattern matching using ART1, which can learn and recognize the binary palm vein pattern. This ART1 matching module can be constructed by an attentional subsystem and an orienting subsystem. The attentional subsystem is responsible for competitive learning and enhancing the palm vein pattern by suppressing noise. The orienting subsystem can work as a novelty detector. Initially the bottom-up and top- down weights of ART1 can be set as

$$
\begin{aligned}
& B_{y}=L(L-1+M) \\
& T_{y}=1
\end{aligned}
$$

where $\mathrm{L}$ is the number of layers and $\mathrm{M}$ is the number of users. When the vigilance level is satisfied the bottom-up and topdown weights can be calculated by

$$
\begin{aligned}
& B_{\mathrm{g}}=2 \times T_{\mathrm{g}} \times x_{t / 1}+s t_{\mathrm{g}} \times x_{s} \\
& \left.T_{\mathrm{g}}=T_{\mathrm{g}} \text { (old }\right) \times x_{s}
\end{aligned}
$$

where $x i$ is the element in the input vector.

\section{FUSION AT DECISION LEVEL}

The overall decision is simply the fusion of these classifiers using Naive Bayes fusion approach. The Naive Bayes (NB) fusion approach considers decisions of the individual classifiers are independent. Using $\mathrm{n}$ features $\mathrm{f}$ (individual classifier decisions) that picks a particular decision $\mathrm{d}$ of a set of Decisions D, the NB decision rule is classify $\left(f_{1} \ldots f_{n}\right)$ arg $\max _{\mathrm{e}} p(D=d) \prod_{i=1}^{n} p\left(F_{i}=f_{i} / D=d\right)$

\section{RESULTS AND DISCUSSION}

The proposed multimodal palm vein recognition is carried out on 5 samples of 50 users. Palm vein reader based on NIR imaging technique obtains the palm vein images. The following steps do the palm vein recognition. In this paper image de-noising and enhancement is performed by GSZ shock filter. The real part of the GSZ shock filter provides the de-noised and enhanced image. The results of median filter and various shock filters are compared in terms of SNR (Signal to Noise Ratio) of de-noised image which can be shown in table 1.

In figure 3 shows that GSZ shock filter can be improved the SNR better than the other filters, more visually appealing and to discontinuities in Palm vein images can be adapted.

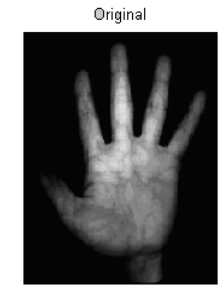

A-M
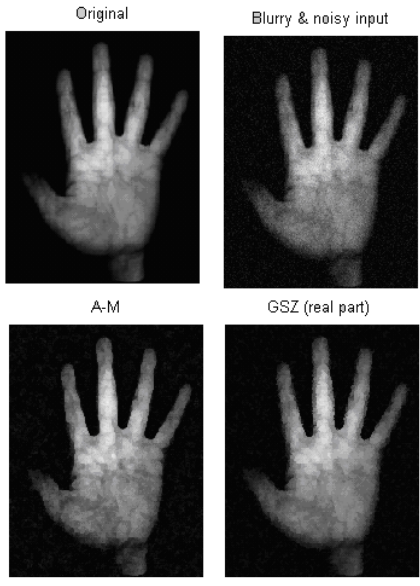

GSZ (real part)
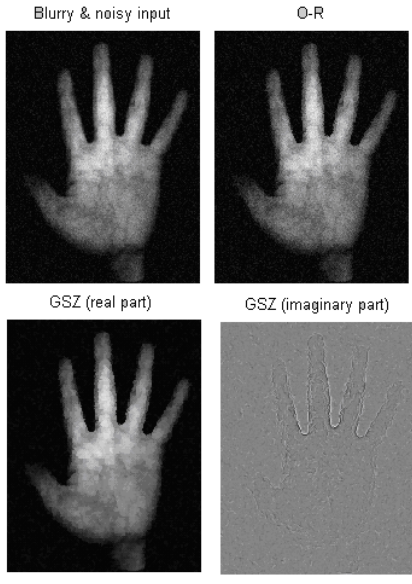

GSZ (imaginary part)

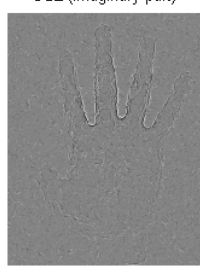

\begin{tabular}{|c|c|c|c|c|c|}
\hline 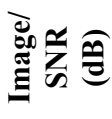 & 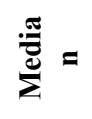 & 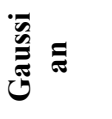 & ठै & $\sum$ & 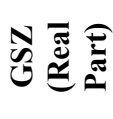 \\
\hline $\begin{array}{c}\text { Palm } \\
\text { Vein } \\
\text { Image }\end{array}$ & 9.1358 & 11.2999 & 13.5321 & 15.7612 & 17.7041 \\
\hline
\end{tabular}

Figure 3. Preprocessed Palm Vein Images

Table 1.Comparison result of filtering techniques

The hand shape feature is extracted from the palm vein image, and the fusion is based on a combination by convolution of the hand geometrical features and the palm texture to construct the multiple features set. The palm texture characteristics are extracted by a Gabor filter. The hand shape feature, palm texture and the convolved multiple feature set are shown in fig. 4 .

The palm vein features such as texture and shape with directional information are extracted for recognition. The direction information is extracted by performing skeletonization and the template used by S. Malki et al [10]. The shape feature is extracted by the contour imaging. Skeleton of the palm vein can be extracted by applying thinning and pruning algorithms. Bifurcation and ending points can be extracted from the skeleton of the palm vein. The various extracted features are shown in Fig 5 - Fig 8.

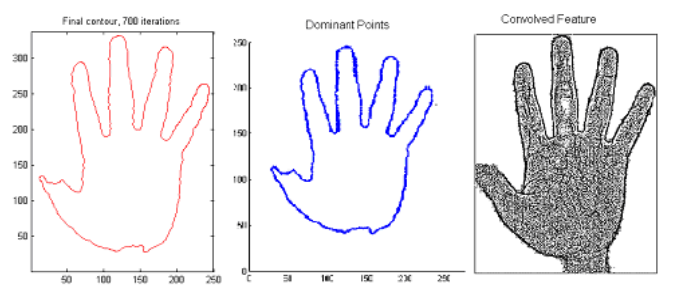

Figure 4. Shape, texture and convolved feature set 
Automatic subset selection is required to select the optimal feature subset. In this paper Adaptive sequential floating forward search (ASFFS) approach is considered to extract salient features from the multiple features set. The optimized convolved multiple feature set, skeleton, bifurcation and ending points are shown in figure 9 .

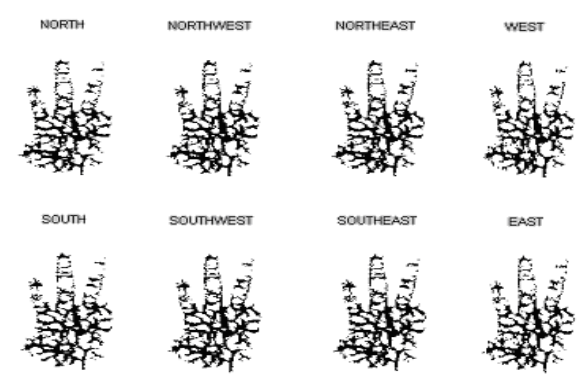

Figure 5. Binary image in eight directions

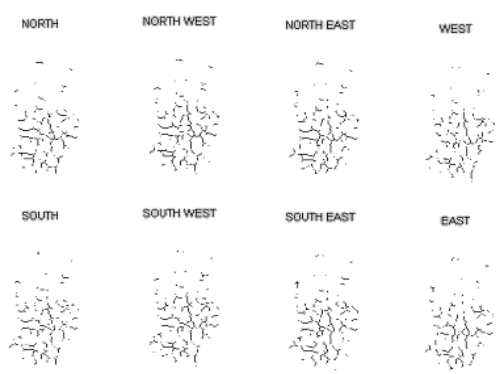

Figure 6. Skeleton of palm vein in eight directions

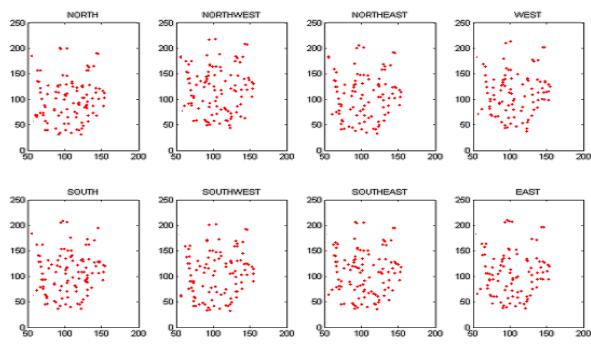

Figure 7. Bifurcation points in eight directions

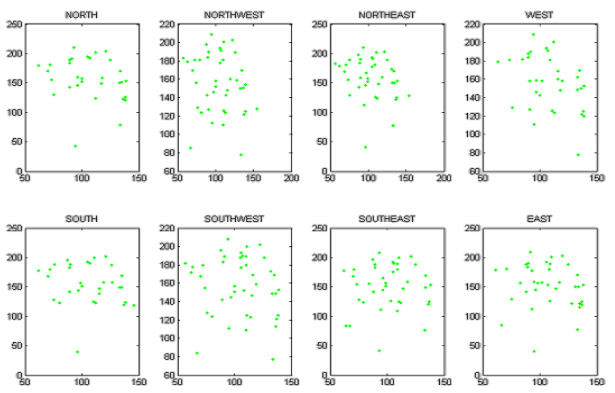

Figure 8. Ending points in eight directions
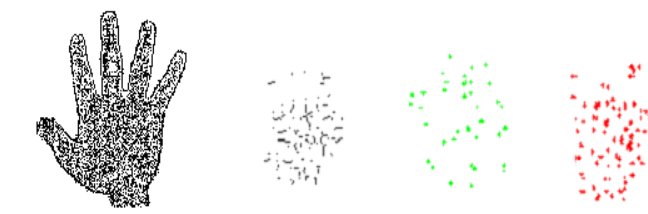

Optinal Convolved feature

Optimal Skeleton Optinal Bifurcation

Optimal Ending Points

Figure 9. Optimal Feature set

\begin{tabular}{|c|c|c|c|c|}
\hline \multicolumn{5}{|c|}{ Table 2 . Performance comparison of unimodal and } \\
multimodal palm vein recognition
\end{tabular}

Table 3 : Performance comparison of unimodal and multimodal palm vein recognition

\begin{tabular}{|l|c|c|c|c|}
\hline \multicolumn{1}{|c|}{ Approach } & NFR & NEVA & FRR & RA \\
\hline Unimodal & 980 & $10^{5}$ & 0.0098 & $99 \%$ \\
\hline Multimodal & 400 & $10^{5}$ & 0.004 & $99.6 \%$ \\
\hline
\end{tabular}

Table 4. Performance comparison of Laplacianpalm and Multimodal palm vein recognition

\begin{tabular}{|l|l|l|l|l|}
\hline Approach & $\begin{array}{c}\text { Fusion } \\
\text { Type }\end{array}$ & $\begin{array}{l}\text { No. of } \\
\text { features } \\
\text { selected }\end{array}$ & $\begin{array}{l}\text { MER } \\
\%\end{array}$ & $\begin{array}{l}\text { RA } \\
\%\end{array}$ \\
\hline Laplacianpalm & $\begin{array}{l}\text { Imaging } \\
\text { Level }\end{array}$ & 27 & 1.016 & 98.984 \\
\hline Multimodal & $\begin{array}{l}\text { Extraction } \\
\text { and } \\
\text { Decision } \\
\text { Level }\end{array}$ & 16 & 0.4 & 99.6 \\
\hline
\end{tabular}

This comparative experiment is conducted on 5 samples of 50 users. The ART1 neural network can be trained by the samples of 25 users and tested by the samples of all 50 users. Fusion at decision level can be done by Naïve Bayes fusion approach with the higher priority value 3 assigned to convolved feature set, the next priority value 2 assigned to skeleton of the vein image and the lower priority value 1 assigned to both bifurcation and ending points of the vein pattern. These priority values can be obtained by trial and error basis. In Table 2, NFA is Number of False Attempts, NIVA is Number of Imposter Verification attempts, NFR is Number of False Rejections and NEVA is Number of Enrollee Verification attempts. 
Table 5: Execution Time Calculation for Laplacianpalm vein recognition system (5 samples of each user)

\begin{tabular}{|l|r|}
\hline Process Type & Execution Time (ms) \\
\hline Preprocessing & 40 \\
\hline Image registration & 100 \\
\hline Fusion (Imaging level) & 310 \\
\hline Feature Extraction & 18 \\
\hline Pattern Matching & $9(\mu \mathrm{s})$ \\
\hline Total Execution Time & 468 ms and 9 $\boldsymbol{\mu s}$ \\
\hline
\end{tabular}

Table 6: Execution Time Calculation for Multimodal palm vein recognition system considering fusion at decision level

\begin{tabular}{|l|r|}
\hline Process Type & Execution Time (ms) \\
\hline Preprocessing & 5 \\
\hline Feature Extraction & 17.8529 \\
\hline Optimization (ASFFS) & 18.4219 \\
\hline $\begin{array}{l}\text { Fusion at Extraction Level } \\
\text { (Convolution) }\end{array}$ & 6.534 \\
\hline Pattern Matching (ART1) & 6.5242 \\
\hline Decision & 0.17388 \\
\hline $\begin{array}{l}\text { Fusion at Decision Level } \\
\text { (Navie Bayes) }\end{array}$ & 2.5454 \\
\hline $\begin{array}{l}\text { Total Execution Time } \\
\text { (Single sample of each } \\
\text { user) }\end{array}$ & $\mathbf{5 7 . 1 1 4 7 8}$ \\
\hline $\begin{array}{l}\text { Total Execution Time } \\
\text { (5 samples of each user) }\end{array}$ & $\mathbf{2 8 5 . 5 7 3 9}$ \\
\hline
\end{tabular}

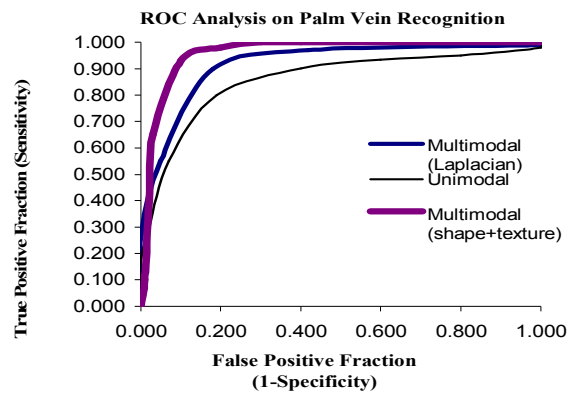

Figure 10. ROC Analysis of palm vein recognition systems

The comparison of performance analysis of Unimodal with multimodal and Unimodal with Laplacianpalm are shown in Table 2-4. When compare to "Laplacianpalm", this proposed multimodal palm vein recognition system can perform efficient authentication. The performance comparisons of these methods are done by ROC analysis. The overall ROC performance of unimodal is $\mathrm{Az}=0.92$, Laplacianpalm is $\mathrm{Az}=0.945$, with comparison to ASFFS and LDA is $\mathrm{Az}=0.993$ which shown in Figure10. The execution times of these palm vein recognition systems are analyzed. The proposed hybrid palm vein recognition system works faster than the Laplacianpalm multimodal palm vein recognition system, which is observed from Table 5 and Table 6.

\section{CONCLUSION}

The palm vein recognition system using multilevel fusion of multimodal features and neural network classifier has been developed. The shape and texture features have been extracted and multimodal features have been obtained at feature extraction level as well as matching score level. The Neural network classifier has been used to classify the vein patterns for making necessary decision. It is concluded from the analysis that the multimodal palm vein recognition system provides better performance compared unimodal features.

\section{REFERENCES}

[1]. Fujitsu Computer Products of America, Inc., www.fcpa.fujitsu.com -White Paper -"Palm Vein Authentication Technology".2006.

[2]. L.Wang and C.G Leedham, "A Thermal Hand Vein Pattern Verification System", In Lecture Notes in Computer Science, Springer, 2005.

[3]. Jian-Gang Wang, Wei-Yun yau, Andy Suwandy, "Fusion of Palmprint and palm vein images for person recognition based on "laplacianpalm" feature", 1-4244-1180-7/07/ IEEE- 2007.

[4]. Jian-Gang Wang, Wei-Yun yau, Andy Suwandy, "Feature Level Fusion of Palmprint and palm vein for person recognition based on a "Junction Point" Representation ", 978-1-4244-17643/08/IEEE- 2008.

[5]. Zhongli Wang, Baochang Zhang, Weiping Chen, Yongsheng Gao, "A performance Evaluation of Shape and Texture based methods for vein recognition", DOI 10.1109/CSIP.2008.106 - 978-0-76953119/08- IEEE - 2008.

[6]. Chih-Lung Lin, Kuo-Chin Fan, "Biometric Verification Using Thermal Images of PalmDosra Vein Patterns", IEEE Transactions on Circuits and Systems for Video Technology, Vol.14, No.2, Feb 2004.

[7]. Weickert. J. "Coherence-enhancing Diffusion Filtering", International Journal of Computer Vision, Vol. 31(2/3), Pages 111-127, 1999.

[8]. Guy Gilboa, Nir Sochen and Yehoshua Y. Zeevi, "Image Enhancement and Desnoising by Complex Diffusion Processes", IEEE Transactions on Pattern Analysis and Machine Intelligence, Vol.26, No.8, 2004.

[9]. Guy Gilboa, Nir Sochen and Yehoshua Y. Zeevi, "Regularized Shock Filters and Complex Diffustion", Springer - Verlag Nerling Heidelberg, ECCV 2002, LNCS 2350, pp 399-413, 2002.

[10]. Suleyman Malki, Yu Fuqiang, Lambert Spaanenburg, "Vein Feature Extraction Using DT-CNNs", International Workshop on Cellular Neural Networks and Their Applications, IEEE 2006. 
[11]. Julien Doublet, Olivier Lepetit, Marinette Revenu, "Contact less Hand Recognition Using Shape and Texture Features", ICSP2006 Proceedings.
[12]. Somol. P, Pudil. P, Novovicova. J, Paclik. P, "Adaptive Floating Search Methods in Feature Selection", Pattern Recognition Letters 20, 1157 - 1163, 1999. 